\title{
BeliefBank: Adding Memory to a Pre-Trained Language Model for a Systematic Notion of Belief
}

\author{
Nora Kassner ${ }^{1,2}$, Oyvind Tafjord ${ }^{1}$, Hinrich Schütze ${ }^{2}$, Peter Clark ${ }^{1}$ \\ ${ }^{1}$ Allen Institute for AI, Seattle, WA \\ ${ }^{2}$ Center for Information and Language Processing, LMU Munich, Germany \\ kassnerdcis.lmu.de \\ \{oyvindt, peterc\} eallenai.org
}

\begin{abstract}
Although pretrained language models (PTLMs) contain significant amounts of world knowledge, they can still produce inconsistent answers to questions when probed, even after specialized training. As a result, it can be hard to identify what the model actually "believes" about the world, making it susceptible to inconsistent behavior and simple errors. Our goal is to reduce these problems. Our approach is to embed a PTLM in a broader system that also includes an evolving, symbolic memory of beliefs - a BeliefBank - that records but then may modify the raw PTLM answers. We describe two mechanisms to improve belief consistency in the overall system. First, a reasoning component $-\mathrm{a}$ weighted MaxSAT solver - revises beliefs that significantly clash with others. Second, a feedback component issues future queries to the PTLM using known beliefs as context. We show that, in a controlled experimental setting, these two mechanisms result in more consistent beliefs in the overall system, improving both the accuracy and consistency of its answers over time. This is significant as it is a first step towards PTLM-based architectures with a systematic notion of belief, enabling them to construct a more coherent picture of the world, and improve over time without model retraining.
\end{abstract}

\section{Introduction}

Intelligent agents are typically considered to have beliefs about the world - propositions that they take as true (Genin and Huber, 2021). In general, a system can be said to (appear to) believe a proposition $p$, e.g., "eagles are birds", if it produces answers consistent with $p$ (and its other beliefs). Pragmatically, we expect the system to (a) give a consistent answer to different paraphrases of the question " $p$ ?" ("Are eagles birds?", "Is an eagle a type of bird?", ...), and (b) give correct answers about implications of $p$ ("Eagles lay eggs", "Eagles have feathers", ...),

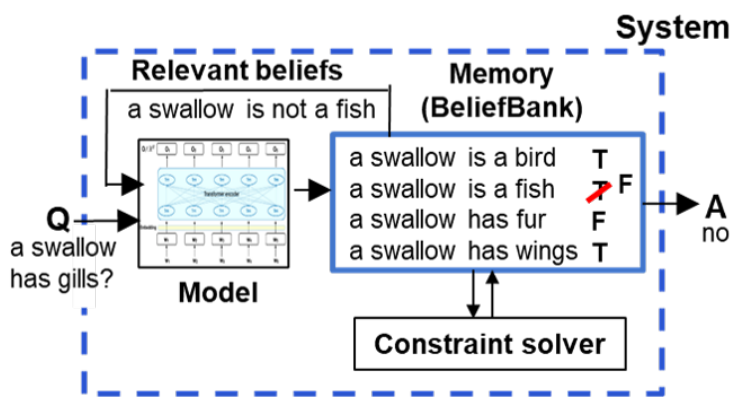

Figure 1: The proposed architecture. The model's raw answers are stored in a persistent, symbolic memory (BeliefBank), and two mechanisms attempt to improve them: (a) A constraint solver flips beliefs (e.g., the belief that "a swallow is a fish") that clash significantly with others. (b) A feedback mechanism poses new questions using existing, relevant beliefs (e.g., "a swallow is not a fish") as the query context. We find that both consistency and accuracy of the overall system improve. Example: The model $M$ shown in the figure incorrectly answers "yes", when asked "a swallow has gills?". But (as shown above) if reminded of its previous answer "a swallow is not a fish", $M$ correctly answers "no".

conditional on its other knowledge and reasoning abilities.

Maintaining a consistent set of beliefs (a "belief system") is a key facet of intelligence, as it can help debug errors and encourage rational behavior. However, although PTLMs contain substantial world knowledge (Petroni et al., 2019), their answers to probing questions can be inconsistent (Elazar et al., 2021; Kassner and Schütze, 2020), even after specialized training to reduce inconsistency (Ribeiro et al., 2019; Li et al., 2019). As a result, it is sometimes hard to pin down what a PTLM actually "believes", making them susceptible to inconsistent and/or irrational behavior. Our goal is a first step to avoid these problems by embedding a PTLM in a broader system with a clearer notion of belief (see Figure 1).

Prior work in AI, including in formal logic (Genesereth and Nilsson, 1987), belief mainte- 
nance (De Kleer, 1986; Dechter and Dechter, 1988), and uncertainty (Pearl, 1986), offers models for how beliefs can be managed. Most importantly, it posits that creating a coherent set of beliefs a kind of "mental model" of the world (JohnsonLaird, 1983) - is a constructive process requiring explicit representation of beliefs, and inference about their dependencies. Based on this, our approach is to embed a PTLM in a broader system with an evolving, symbolic memory of beliefs - a BeliefBank - along with two mechanisms to improve belief consistency of the overall system. First a reasoning component - a weighted MaxSAT (satisfiability) solver - reasons about belief dependencies and revises beliefs that significantly clash with others. Second, a feedback component poses future queries to the model using known beliefs as context, aiming for more accurate and consistent answers from the PTLM itself. The BeliefBank represents the overall system's beliefs (a "mental model") about the world, constructed by deliberating over the noisy output of a raw PTLM.

We explore this in a controlled experimental setting where both candidate beliefs and constraints between them are provided. Candidate facts are simple sentences that may be true or false, e.g., "An eagle is a bird" (T), "An eagle is a mammal" (F). Constraints are between (variabilized) facts, e.g., " $\mathrm{X}$ is a bird $\rightarrow \mathrm{X}$ has wings". These allow us to both probe and measure improvement in the consistency and accuracy of a system's beliefs, compared with an original PTLM.

In contrast to prior work, this system does not rely on fine-tuning the PTLM. Fine-tuning requires expensive training data, and risks destabilizing the model's performance on other tasks outside the scope of training. Instead, our system functions without training data, explicitly reasoning about beliefs using an external mechanism, thus allowing both controllability and interpretability. Most significantly, we find that improving consistency in this way improves accuracy, while earlier finetuning-based approaches report either no accuracy gains (Ribeiro et al., 2019; Minervini and Riedel, 2018; Li et al., 2019) or only slight gains (Asai and Hajishirzi, 2020).

We make the following contributions:

1. We show that a PTLM-based system can be given a consistent notion of belief by augmenting the PTLM with a global memory the BeliefBank - that can be deliberated over.
Specifically, we show that two mechanisms constraint reasoning and feedback - improve the overall system's accuracy and consistency over time.

2. We contribute a targeted dataset to measure a system's consistency against given constraints.

3. We provide an analysis of the failure modes and directions for future work.

This work is significant as it is a first step towards PTLM-based architectures that have a systematic notion of belief, allowing them to construct a more coherent picture of the world, and improve over time without model retraining. ${ }^{1}$

\section{Related work}

The idea that agents should have a belief system dates back to the earliest years of AI, e.g., McCarthy (1959) envisioned representing a system's beliefs as formal propositions along with a reasoning process to identify what follows. Multiple subfields of AI have explored ways of representing and updating beliefs, including in formal logic (Genesereth and Nilsson, 1987; Moore, 1983), belief revision (De Kleer, 1986; Dechter and Dechter, 1988), and uncertainty (Pearl, 1986). Similarly, work in cognitive science has promoted mental models - coherent, constructed representations of the way the world is believed to be - as central to understanding and communication (Johnson-Laird, 1983; Gentner and Stevens, 1983; Hilton, 1996). We draw on these ideas, proposing how they can be layered on top of PTLMs, here representing beliefs as NL statements rather than formal structures.

Although PTLMs contain extensive world knowledge (Petroni et al., 2019; Roberts et al., 2020), they can be inconsistent in their answers to probing questions (Ettinger, 2020; Davison et al., 2019; Ravichander et al., 2020; Elazar et al., 2021; Subramanian et al., 2020), making their "world model" unclear. Although various approaches have improved answer consistency, mainly through modified model training, e.g., (Ribeiro et al., 2019; Minervini and Riedel, 2018; Li et al., 2019; Asai and Hajishirzi, 2020), they have not solved the problem. Current PTLMs still behave as a source of noisy knowledge, rather than projecting a coherent picture of the world (Kassner and Schütze, 2020).

A close analogy to our task is in knowledge graph (KG) construction. Pujara et al. (2013)

\footnotetext{
${ }^{1}$ Dataset is available at https://allenai.org/data/beliefbank
} 
define "knowledge graph identification" as the task of building a maximally consistent $\mathrm{KG}$ given noisy candidate facts and their extraction confidences, and constraints between them. They develop a solution using probabilistic soft logic (PSL) (Broecheler et al., 2010) as their constraint reasoner. Our reasoning component follows similar ideas, but applied to the noisy predictions of a PTLM. On the face of it, it is not clear how to plug a constraint solver into a PTLM, given their very different natures. Our solution introduces a global persistent memory, making this novel combination of technologies possible. To our knowledge this has not been done before.

Our work presents a broader system architecture in which a PTLM is embedded, along with a dynamic, persistent memory. While there are prior neural architectures that include an associated memory, e.g., (Henaff et al., 2016; Sukhbaatar et al., 2015; Graves et al., 2016), these components typically play the role of a short-term working memory to help computation. In contrast, our BeliefBank is a persistent, long-term memory, and we treat the PTLM as a component within a larger system. Our work also differs from retrieval-augmented architectures, e.g., RAG (Lewis et al., 2020), REALM (Guu et al., 2020), that augment model input with external retrievals. Rather, our memory is reflective, built from model outputs and reasoned over.

Our feedback mechanism uses old answers to help answer new questions. This builds on prior work such as Self-Talk (Shwartz et al., 2020), where a model asks itself related questions to help with new answers, and can be seen as a form of dynamic prompt engineering (Liu et al., 2021). In our case, feedback is selected from a global BeliefBank, rather than generated with templated subqueries, potentially allowing more control over feedback selection.

Finally, our system performs a kind of continual learning (Parisi et al., 2019; Carlson et al., 2010). While recent work in this area has focused on dynamic update of model parameters, e.g., (Ebrahimi et al., 2021), our work leaves the model fixed, and seeks improvement in the broader system in which the model is embedded, exploring an alternative and potentially more interpretable architecture towards this goal.

\section{Task}

Our goal is to ascribe a clearer notion of "belief" to a system that includes a model $M$, by improving, over time, the consistency and accuracy of its answers (compared with $M$ ) to a stream of questions. We measure this with true/false probing, where we are also given a set of constraints between answers: Given:

- a stream of sentences $Q$, interpreted as questions to the model

- a set of constraints $C(S)$ between (the truth values of) sentences in $Q$, each annotated with a weight $w_{i}$ (A penalty $w_{i}$ is applied if $c_{i} \in C(S)$ is violated)

- a model $M$ that takes as input a question $q \in Q$ and optionally an (NL) context $X$ (consisting of answers to previously posed questions), and predicts a True/False answer $A$ with confidence score $F$

\section{Accumulate:}

- the True/False labels for $Q$ predicted by $M$, optionally corrected by the constraint solver, so as to maximally improve accuracy (with respect to gold labels) and consistency (minimize total penalties of constraint violations)

\section{Approach}

Our approach adds a memory layer, called the BeliefBank, on top of the model to globally track beliefs. Two mechanisms are then used to manage the BeliefBank beliefs, namely constraint reasoning and feedback, as we now describe.

\subsection{Definitions}

Let

- a belief $b_{i}$ be a triple $\left(s_{i}, l_{i}, w_{i}\right)$, where

- $s_{i}$ is a sentence $\in S$

- label $l_{i} \in\{\mathrm{T}, \mathrm{F}\}$ denotes the system's True/False belief about the truth of $s_{i}{ }^{2}$

- weight $w_{i}$ is a number $\in[0,1]$ representing the system's strength of that belief

For example:

("a poodle is a dog", T, 0.9)

denotes the belief (strength 0.9 ) that "a poodle is a dog" is a true statement (T).

\footnotetext{
${ }^{2}$ Strictly, the label $\mathrm{F}$ denotes the belief that the negation of $s_{i}$ is true, e.g., ("a poodle is a bird",F,...) denotes the belief "a poodle is not a bird".
} 
- a BeliefBank $B(S)=$ a set of beliefs over sentences $S=s_{1}, \ldots, s_{n}$

- a constraint $c_{i}=$ a 5-tuple of the form $\left(s_{i} . l_{i} \rightarrow s_{j} . l_{j}, w_{i}\right)$ where

- $s_{i}, s_{j}$ are sentences $\in S$,

- $l_{i}, l_{j} \in\{\mathrm{T}, \mathrm{F}\}$. If $s_{i}$ has truth value $l_{i}$, denoted $s_{i} \cdot l_{i}$, then $s_{j}$ is expected to have truth value $l_{j}$, denoted $s_{j} . l_{j}$.

- $w_{i}$ denotes the strength of that expectation (a penalty $w_{i}$ is applied if violated).

For convenience, a shared variable $\mathrm{X}$ can be used in $s_{i}, s_{j}$, allowing a set of grounded constraints to be expressed in one statement, e.g.,

("X is a dog".T $\rightarrow$ "X has a tail".T, 0.8 )

expresses that if something is a dog, then it should (T) have a tail, with a penalty of 0.8 applied if it does not. Mutual exclusivity is expressed using two rules, e.g., that fish and birds are mutually exclusive is expressed:

("X is a bird". T $\rightarrow$ " $X$ is a fish".F, 1.0)

("X is a fish".T $\rightarrow$ "X is a bird".F, 1.0)

where " $F$ " indicates the conclusion should be false if the condition here is true $(\mathrm{T})$.

- a constraint graph $C(S)=$ a set of constraints $c_{i}$ over sentences $S$

Given a set of beliefs $B(S)$ about $S$ and a set of constraints $C(S)$, we measure consistency using (the complement of) Li et al. (2019)'s conditional constraint violation $(\tau)$ metric, namely the fraction of constraints whose condition $s_{i} . l_{i}$ is believed, but whose conclusion (that $s_{j}$ has truth value $l_{j}$ ) is not. In other words, over all constraints $c_{i} \in C(S)$, inconsistency $\tau$ is

$$
\tau=\left|\left\{c_{i} \mid \neg\left(s_{i} . l_{i} \rightarrow s_{j} . l_{j}\right)\right\}\right| /\left|\left\{c_{i} \mid s_{i} . l_{i}\right\}\right|
$$

i.e., the size of the set of violated constraints $\left(s_{i} . l_{i} \rightarrow s_{j} . s_{j}\right.$ is false) divided by the size of the set of applicable constraints. We then define:

$$
\text { consistency }=1-\tau
$$

\subsection{Methods}

We consider our system in a dynamic setting, where it receives a stream of questions and gradually builds up a BeliefBank of answers (including revising earlier answers). We evaluate two methods for improving the BeliefBank's accuracy and consistency over time:

Constraint solving: Given a model $M$ 's raw answers (with confidences), a constraint solver seeks to reduce constraint violations by potentially flipping answers that maximally clash with other answers.
Feedback: Given a new question $q$, selected beliefs in the BeliefBank are provided as context to $M$ to help it answer $q$ correctly.

Figure 1 shows these components.

\subsubsection{Constraint Solving}

Given a set of beliefs and constraints, the constraint solver has two competing objectives: (a) flip beliefs so as to minimize constraint violations (b) don't flip beliefs, so as to preserve the model's raw answers, i.e., minimize conflict between the model and BeliefBank. To implement this tradeoff, the model's answers are themselves treated as just another constraint, e.g., the answer that "a poodle is a dog" is true (confidence 0.9) is treated as a constraint "a poodle is a dog", with penalty 0.9 if it is violated. To balance the two objectives (a) and (b), the model confidences are scaled by a learned hyper-parameter $\lambda$, trained on a calibration part of our dataset, disjoint from the data then used in experiments (Section 5).

To implement constraint solving, we translate the task into a weighted MaxSAT (satisfiability) problem $P$, for which efficient algorithms with guarantees exist. Each belief becomes a weighted assertion in $P$, e.g., the belief ("a poodle is a dog", $\mathrm{T}, 0.9$ ) is expressed in SAT syntax:

\section{9 "a poodle is a dog"3}

while the constraint ("a poodle is a dog".T $\rightarrow$ "a poodle has a tail".T, 0.8 ) is expressed:

\section{8 "a poodle has a tail" -"a poodle is a dog"} (literally: "a poodle has a tail" OR NOT ("-") "a poodle is a dog"). We then apply the solver Z3 (De Moura and Bjørner, 2008) to $P$, which outputs a set of truth assignments for all individual sentences in $P$ so as to minimize the weighted sum of violations. If the truth of any sentence has changed, the BeliefBank is correspondingly updated.

\subsubsection{Feedback}

Feedback involves asking the model a question, but with the benefit of knowing answers to prior, related questions. To use these answers in the query, selected beliefs are added to the query context before asking the model. (Note that the selected beliefs are not guaranteed to be correct, of course). Our conjecture is that if the model is explicitly reminded of relevant beliefs when answering a new question, it will answer the question more accu-

\footnotetext{
${ }^{3}$ In practice, strings are replaced with numeric identifiers in SAT syntax, but for clarity we leave them as strings here.
} 
rately and consistently. For example, in Figure 1, when asked "Do swallows have gills?", our model $M$ incorrectly answers "yes". But if reminded that swallows are not fish, by asking: "CONTEXT Swallows are not fish. QUERY Do swallows have gills?" the model now correctly answers "no".

We evaluate two policies for choosing which beliefs to feed back to $M$ when asking question $q$ about entity $e$ :

1. on topic beliefs, namely current beliefs about entity $e$, randomly selected from the BeliefBank

2. most relevant on topic beliefs (i.e., again about $e$ ), using the constraint graph to identify relevance. As the constraint graph captures potential clashes that the answer to $q$ could cause, we use the graph to identify beliefs that would be most affected by that answer. For example, if the current query is: "Is a poodle an animal?", the constraint graph identifies potential clashes that would occur if the model answered "yes", and also clashes if it answered "no". Here, if the model answered "no", the resulting belief ("a poodle is not an animal") would strongly clash with other beliefs "A poodle is a dog." and "A poodle is a mammal.", so these two are strong candidates for the context. We select the three strongest clashing beliefs found in this way, considering both "yes" and "no" answers to $q$. If no relevant fact is present, we use a randomly selected topic belief instead.

In both cases, three beliefs are selected, this number was empirically found to be most effective.

\section{Dataset}

We create a dataset ${ }^{4}$ to test our approach in a controlled way, allowing us to perform systematic experiments to evaluate behavior. The dataset contains two parts, constraints and facts, defined over simple sentences such as "a swallow is a bird."

\subsection{Constraints}

The dataset contains two kinds of constraints:

positive implications: conclusion truth value $1_{j}=$ $\mathrm{T}$ (true), e.g.,

" $X$ is a dog. $T \rightarrow$ " $X$ has a tail.". $T$

mutual exclusivities: expressed as a pair of constraints with $\mathrm{l}_{j}=\mathrm{F}$ (false), e.g.,

\footnotetext{
${ }^{4}$ Dataset is available at https://allenai.org/data/beliefbank
}

"X is a dog".T $\rightarrow$ "X is a bird.”. $F$
"X is a bird". $T \rightarrow$ "X is a dog.".F

expresses that an entity cannot be both a dog and a bird at the same time.

Positive implications were manually gathered from ConceptNet (Speer et al., 2017). First, we identified 121 general concepts of interest, e.g., "mammal", then converted selected triples about them to constraints (Details of the selection process are in Appendix A). For example, the ConceptNet triple (dog,HasA,tail) becomes the constraint " $\mathrm{X}$ is a dog" $\rightarrow$ "X has a tail". We also add weaker, disjunctive constraints in the backward direction, e.g., "X has a tail" $\rightarrow$ "X is a $\operatorname{dog}$ " OR "X is a cat" OR .... for all entities with tails. Mutual exclusivities were gathered from the "isa" taxonomies in ConceptNet and WordNet (Fellbaum, 2005), using the approximation that siblings in the noun hierarchy are mutually exclusive. Thus, for any pair of siblings, we add a mutual exclusivity constraint (using two constraint rules).

We collected 2612 constraints in this fashion (1836 forward implications, $2 * 388$ bidirectional mutual exclusivities).

\subsection{Constraint Weights}

Constraint weights need to be set appropriately to mix well with the model's confidences inside the weighted SAT solver. We use a development set of 1072 facts about seven entities to set one constraint weight for the forward direction of the implications and the mutual exclusivity rules and a second one for the backward direction of the implications. To do this we perform a grid search over these parameters, finding the values that result in the highest F1 (accuracy) after running the constraint solver over the raw model's beliefs about these facts.

\subsection{Facts}

We also collect a set of truth-labeled facts about different entities, relevant to the constraints. To do this, we select a new entity, e.g., "poodle", that is a member of one of our general concepts, e.g., "dog", then instantiate the constraint graph with that entity (i.e., set $X=$ "poodle"). We then identify the leaf (source) nodes of that graph, just considering forward implication rules, i.e., finding facts not implied by other facts in the graph, and manually annotate their True/False labels. We then use the implications and mutual exclusivities to infer other True/False labels for other sentences, i.e., we propagate the annotated labels through the graph. This 
provides "silver" labels for sentences reachable in this way (a subset of all the sentences in the graph) - silver because the implications are soft, hence not guaranteed to hold for all entities.

We repeat this for 85 entities (animals and plants), resulting in a final dataset containing 12,525 "silver" facts (sentences + True/False labels). Note that this data is purely for evaluation. There is no training phase or training data. The system does not have access to any labeled data besides the constraint rules.

\section{Model}

The fixed model $M$ that we use for our experiments is Macaw (Tafjord and Clark, 2021), a state-of-theart T5 QA model fine-tuned on $\approx 400 \mathrm{k}$ QA pairs. To query the model, we pose the query (optionally with a textual context), and let the model choose between the two answer options "yes" and "no". The model also outputs an answer confidence, used as the belief weight.

We use the T5-large version of this model. Note that we do not retrain the model for this work; rather, it is used as a black-box QA module in the broader system (Figure 1). Other models could equally have been used.

\section{Experiments}

We evaluate our system in a dynamic setting in which it receives a stream of questions, building up and revising a BeliefBank. To simplify the evaluation, we consider questions to arrive in batches, and evaluate the BeliefBank after each batch, measuring accuracy $(\mathrm{F} 1)^{5}$ and consistency (1- $\tau$, Section 4.1) of the BeliefBank so far, comparing with the gold labels. We evaluate four configurations:

Raw model: The BeliefBank simply records the raw model's answers ${ }^{6}$

Constraint-Solving: After each batch, the constraint solver is run over all the (raw) model answers so far, and the BeliefBank updated accordingly.

Feedback: Questions in batch $n$ are posed to the model using a context selected from the be-

\footnotetext{
${ }^{5}$ We measure accuracy with F1 (on the True class) rather than $\%$ correct because the True/False distribution in our dataset is unbalanced, with significantly fewer True than False answers. F1 avoids scores being dominated by negative answers.

${ }^{6}$ To the best of our knowledge there are no other baseline models to compare to as consistency based Q\&A does not go beyond paraphrases and relies on finetuning (Elazar et al., 2021).
}

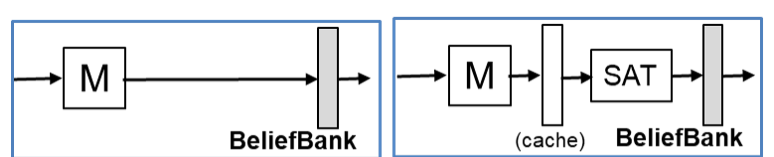

(A) raw model

(B) constraint-solving

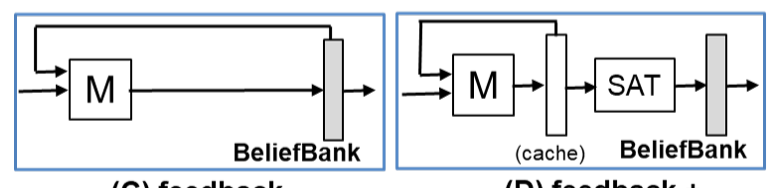

(C) feedback

(D) feedback + constraint-solving

Figure 2: The four configurations we evaluate. In (B), the contraint-solver (SAT solver) is run over all model $\mathrm{M}$ answers so far. In (C), current beliefs are fed back as context for new questions. (D) combines the two.

liefs already in the BeliefBank (batches 1 to $n-1)$. We evaluate two selection strategies:

Feedback (on-topic): Random beliefs about the entity $e$ being queried about

Feedback (relevant): On-topic beliefs (i.e., again about $e$ ) that are most relevant to the query, as defined in Section 4.2.2

Feedback + Constraint-Solving: A combination of the two.

These configurations are illustrated in Figure 2.

\subsection{Results}

The results are shown in Figure 3, showing the changing accuracy and consistency of the growing BeliefBank with time, for different configurations. Each time-step (batch) represents another $10 \%$ of the test questions being posed to the system. (The same data is presented in tabular form in Appendix B). Several conclusions can be drawn:

- Use of feedback, constraint-checking, or both, all result in a continually improving accuracy over time. This is a significant result, showing a larger system can continually improve even if its internal PTLM component is fixed. (The raw accuracy of the PTLM itself is necessarily constant).

- Use of the constraint-solver results in very high $(\mathbf{9 5 \%})$ consistency, indicating that it is doing its job well, and also improving accuracy substantially $(+17 \%$ over the raw model). The constraint-solver has a global view of the BeliefBank, and thus can balance all beliefs seen so far with the provided constraints to make a decision.

- Relevant feedback results in significant consistency gains compared with just on-topic feedback. As relevant beliefs are exactly those that may clash with the answer to the current question (Section 4.2.2), this encourages the model to 

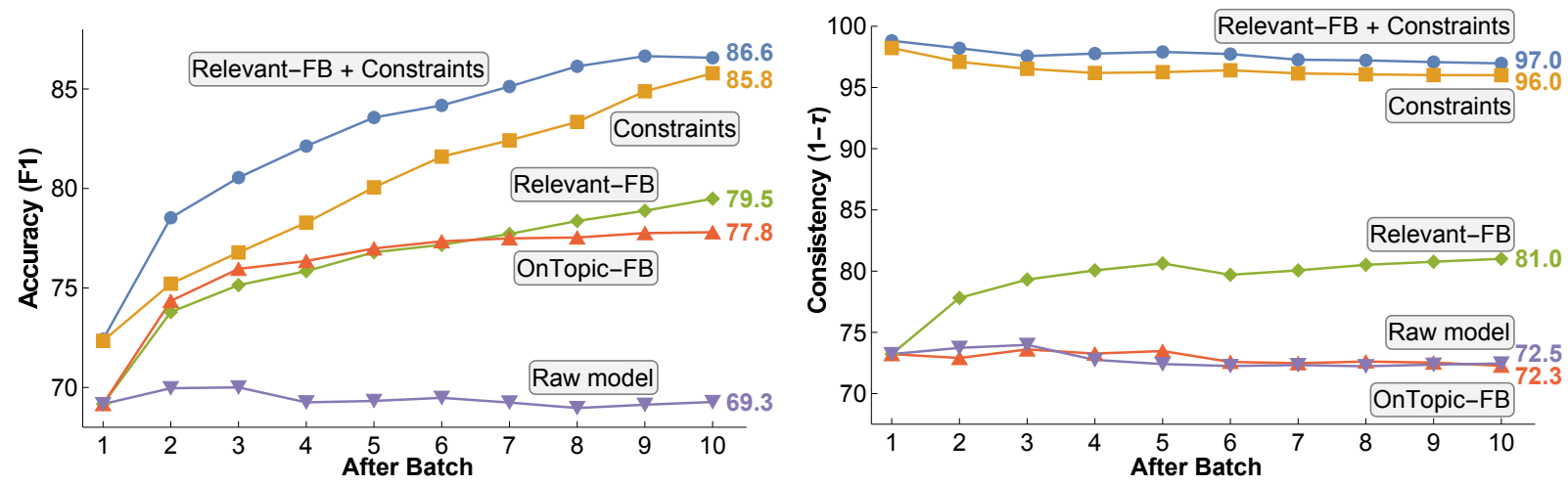

OnTopic-FB = using (randomly selected) on-topic feedback from old answers for new queries.

Relevant-FB = using most relevant on-topic feedback for new queries.

Constraints = running the constraint-solver after each batch.

Figure 3: Accuracy (left) and consistency (right) of the growing BeliefBank, as the system answers incrementally more questions (each batch $=10 \%$ of the queries). Relevant feedback, constraint-solving, and both, all help improve both F1 and Consistency.

answer consistently with those beliefs, promoting consistency. Of course, this could hurt accuracy if those relevant beliefs were wrong, amplifying the errors. In fact, the overall accuracy remains about the same as with on-topic feedback, and significantly better than the model's raw answers.

- The greatest gains are for feedback and constraint-solving combined, resulting in $+18 \%$ F1 (absolute) over the raw model accuracy. This suggests that feedback and constraints can work together in a positive way.

- As a sanity check we also tried using random beliefs about other entities (off-topic) as feedback, but (as expected) the results did not significantly differ from no feedback (raw model), ending at $\approx 72 \% \mathrm{~F} 1$ and $\approx 74 \%$ consistency after batch 10 .

We also evaluated a non-incremental, "omniscient" version of the BeliefBank: Given the raw model answers to all questions, re-ask every question using feedback selected (using relevance) from all the other answers. The resulting accuracy was $74.5 \%$, substantially lower than for the on-topic incremental approaches. Interestingly, this approach's built-in advantage (that every question has access to answers for all other questions) does not outweigh the built-in disadvantage (that those are the raw, rather than incrementally corrected, answers). This is a significant result demonstrating that the positive feedback loop of the incremental approaches can be advantageous, where feedback feeds more accurate beliefs into the BeliefBank, improving future feedback, etc.

\subsection{Failure Analysis}

We now provide some examples of good and bad flips to better understand the behavior of the model.

First, as an illustration of desired behavior, the raw model incorrectly believes that a pine is both a plant (correct) and a vertebrate (incorrect), when queried. However, this violates a mutual exclusivity rule, so the constraint-solver considers flipping one of these. Flipping "pine is a plant" from $\mathrm{T}$ to $F$ would result in numerous other violations, e.g., "pine is a tree" (which the model also believes) would be violated. As a result, it prefers to (correctly) disbelieve "pine is a vertebrate", improving both accuracy and consistency.

From an analysis of the data, we see that the majority of the raw model errors are false positives - the raw model generally answers (almost) all the positive facts correctly (recall is $\approx 98 \%$ ), but mistakenly thinks many negative facts are also true (precision is $\approx 54 \%$ ). These false positives can be rather unusual facts, e.g., "A poodle is a bathroom." (model's answer: True). It is unsurprising that the model knows most of the positive facts, as they are simple statements about common entities ("eagles can fly"), likely seen in pre-training. However, the fact that the model makes (what a person would view as) catastrophic errors when asked more unusual questions, e.g., believing that "a poodle is plant", reveals that the PTLM's grasp of the world is still incomplete and problematic. The constraint mechanism proposed here essentially asks the model to think about its answers and their consequences, so that it can spot problems that the PTLM alone does not see, and repair them. 
The constraint reasoner can also make mistakes, flipping things the wrong way so as to improve consistency, at the expense of accuracy. For example, the raw model correctly believes that "a rat is not a cat". However, the constraint solver then (incorrectly) flips this to "a rat is a cat", because multiple constraints weakly suggest rats are cats given other beliefs ("rats catch mice", "rats have tails",...), which together add up, causing the (incorrect) flip, including overwhelming the strong (but not infinitely strong) constraint that "a rat is not a feline." This illustrates that the constraint mechanism is sensitive to the number of and weights on constraints, even with automatic hyperparameter tuning (Section 5.2).

Similarly, the feedback mechanism is sensitive to question order, especially if the model's early answers are wrong, as the feedback mechanism causes the model to pay extra (sometime disproportionate) attention to earlier context (Kassner and Schütze, 2020). For example, the bad context "A poodle is not a mammal" (from an earlier bad answer) undesirably causes the model to change its answer for "A poodle is a dog" from true (raw model) to false.

Finally, we can only speculate why feedback improves results, in particular, since the feedback consists of facts that came from the model itself (i.e., that it already knows). One explanation is that feedback may help the model focus attention on important facts, e.g., reminding the model that "a swallow is not a fish" should help it realize that "a swallow has gills" is False (Figure 1). In addition, feedback may possibly help resolve some ambiguities, e.g., the feedback "a swallow has wings" helps identify the bird sense of "swallow". Similar advantageous use of feedback was observed in the SelfTalk experiments (Shwartz et al., 2020).

In future work, the feedback mechanism can be improved further by training it to respond more systematically to feedback (similar to (Clark et al., 2020)) and to better balance implicit and explicit knowledge (Talmor et al., 2020), ideally incorporating different levels of confidence.

\section{Future Work}

\subsection{Human in the Loop}

Although our system is autonomous, its incremental setting combined with the explicit representation of beliefs makes it amenable to a human in the loop. In this setting, a human might spot an egre- gious bad belief in the BeliefBank, and forcibly correct it. Then, ideally, this strong positive datapoint would also improve the model's accuracy on other beliefs, both in the BeliefBank and for future questions. As a brief test of this, we allowed a human to correct all bad beliefs (average 6) in the BeliefBank after just the first batch (10\%) of questions, and then continued as before to completion, using the constraint-solving approach. We find that these limited interventions increased both the final F1 and Consistency each by 2\% (absolute) on top of the gains produced by the corrected beliefs themselves. Although preliminary, this suggests that our architecture may have value in an interactive "machine teaching" setting, where the user is supervising and correcting the system, and it continually improves as a result (Zhu, 2015).

\subsection{Towards Deployment}

Although our work has been in a constrained setting (targeted set of relations, entities and constraints), there is a clear development path to deployment in real QA systems to reduce the kind of irrational behavior we have described, such as in this (real) transcript:

(1) Is oxygen colorless? yes

(2) What color is oxygen? blue

(3) What gas do plants produce? oxygen

(4) What color is the gas plants produce? green

The basic components of our architecture provide a framework to help avoid such irrationality. First, (declarative versions of) questions and model answers would be persistently stored in a BeliefBank. Second, on-topic feedback could be selected to help answer new questions using information retrieval over the BeliefBank. Third, given a source of constraints, e.g., a general rule of taxonomic inheritance, ${ }^{7}$ constraint solving could be applied to spot and reduce clashes. This would require a mechanism to identify when a belief satisfies a constraint's condition or conclusion, e.g., a state-ofthe-art textual entailment engine such as CA-MTL (Pilault et al., 2021). A variant of our system could also work without the distinction of model beliefs and constraints: Instead of providing constraints externally we could treat them as beliefs, e.g., query the model for mutual exclusivities "Can an entity be an animal and a plant?" or implications: "Do dogs have tails?" directly. This would run the risk

\footnotetext{
${ }^{7}$ I.e., that the properties of an entity type usually apply to all its subtypes also.
} 
of adding extra noise, but would eliminate the manual effort involved in generating the constraint set, and therefore improve scalability. Together, such developments would pave the way to real-world QA systems that are more consistent and improve over time, rather than remain static.

\subsection{The Broader Research Agenda}

This work only touches on a broader research agenda, namely how to expand work on PTLMs to encompass the cognitive skills of world modeling and deliberative reasoning ("thinking, fast and slow" (Kahneman, 2011)). In this broader agenda, intelligence is not just about opaque questionanswering, but also about constructing mental models that describe how (some aspect of) the world works (Gentner and Stevens, 1983). Although mental models are abstractions (hence are approximate), they add a powerful, systematic component to understanding that should expand its capabilities.

The BeliefBank can be seen as a simple illustration of this broader agenda. A wider pursuit would include a richer notion of a model, perhaps with more structure to model elements than just sentences; more sophisticated means of model construction than just accumulating and resolving answers; and the generation of explanations to convey the deliberative component's behavior, and ultimately interact with a user. Such mechanisms may be symbolic or neural in nature, e.g., (Talmor et al., 2020). Although these issues are beyond the scope of this paper, our work points to this interesting, larger goal for PTLM research, as well as offering a specific mechanism for belief consistency.

\section{Conclusion}

PTLMs can be inconsistent in their answers to probing questions, and can still give (what to a person appear as) naively wrong answers. This work is a first step towards alleviating these problems. By embedding a PTLM within a larger system with a persistent, global memory - the BeliefBank -, a constraint-solver and feedback mechanism, we have shown that the overall system's behavior is more coherent, both in terms of consistency and accuracy. The additional memory layer can loosely be seen as the system's "mental model", a representation constructed from the PTLM's raw answers.

Our experiments were conducted in a restricted (small set of relations, entities and constraints), controlled setting, and further development is needed to scale to larger and more complex tasks. Nevertheless, the work here is significant as it is a first step towards PTLM-based architectures with a globally consistent notion of belief, allowing them to construct a more coherent picture of the world, and continually improve with time.

\section{Acknowledgements}

This work has been funded by the Allen Institute for AI and the European Research Council (\#740516) and by the German Federal Ministry of Education and Research (BMBF) under Grant No. 01IS18036A. The authors of this work take full responsibility for its content.

\section{References}

Akari Asai and Hannaneh Hajishirzi. 2020. Logicguided data augmentation and regularization for consistent question answering. ArXiv, abs/2004.10157.

Matthias Broecheler, Lilyana Mihalkova, and L. Getoor. 2010. Probabilistic similarity logic. In $U A I$.

Andrew Carlson, J. Betteridge, Bryan Kisiel, Burr Settles, Estevam R. Hruschka, and Tom Michael Mitchell. 2010. Toward an architecture for neverending language learning. In $A A A I$.

P. Clark, Oyvind Tafjord, and Kyle Richardson. 2020. Transformers as soft reasoners over language. In $I J$ CAI.

Joe Davison, Joshua Feldman, and Alexander Rush. 2019. Commonsense knowledge mining from pretrained models. In EMNLP.

Johan De Kleer. 1986. An assumption-based tms. Artificial intelligence, 28(2):127-162.

Leonardo De Moura and Nikolaj Bjørner. 2008. Z3: An efficient SMT solver. In International conference on Tools and Algorithms for the Construction and Analysis of Systems, pages 337-340. Springer.

Rina Dechter and Avi Dechter. 1988. Belief maintenance in dynamic constraint networks. University of California, Computer Science Department.

Sayna Ebrahimi, Suzanne Petryk, Akash Gokul, William Gan, J. Gonzalez, Marcus Rohrbach, and Trevor Darrell. 2021. Remembering for the right reasons: Explanations reduce catastrophic forgetting. $I C L R$.

Yanai Elazar, Nora Kassner, Shauli Ravfogel, Abhilasha Ravichander, E. Hovy, Hinrich Schütze, and Yoav Goldberg. 2021. Measuring and improving consistency in pretrained language models. ArXiv, $\mathrm{abs} / 2102.01017$ 
Allyson Ettinger. 2020. What BERT is not: Lessons from a new suite of psycholinguistic diagnostics for language models. Transactions of the Association for Computational Linguistics, 8:34-48.

Christiane Fellbaum. 2005. Wordnet and wordnets In Keith et al. Brown, editor, Encyclopedia of Language and Linguistics, Second Edition. Elsevier, Oxford.

M. Genesereth and N. Nilsson. 1987. Logical foundations of artificial intelligence. Kaufmann.

Konstantin Genin and Franz Huber. 2021. Formal Representations of Belief. In Edward N. Zalta, editor, The Stanford Encyclopedia of Philosophy, spring 2021 edition. Metaphysics Research Lab, Stanford University.

Dedre Gentner and Albert L. Stevens. 1983. Mental Models. Lawrence Erlbaum Associates.

A. Graves, Greg Wayne, M. Reynolds, Tim Harley, Ivo Danihelka, Agnieszka Grabska-Barwinska, Sergio Gomez Colmenarejo, Edward Grefenstette, Tiago Ramalho, J. Agapiou, Adrià Puigdomènech Badia, K. Hermann, Yori Zwols, Georg Ostrovski, Adam Cain, Helen King, C. Summerfield, P. Blunsom, K. Kavukcuoglu, and D. Hassabis. 2016. Hybrid computing using a neural network with dynamic external memory. Nature, 538:471-476.

Kelvin Guu, Kenton Lee, Z. Tung, Panupong Pasupat, and Ming-Wei Chang. 2020. Realm: Retrievalaugmented language model pre-training. ArXiv, abs/2002.08909.

Mikael Henaff, J. Weston, Arthur D. Szlam, Antoine Bordes, and Y. LeCun. 2016. Tracking the world state with recurrent entity networks. In ICLR.

D. Hilton. 1996. Mental models and causal explanation: Judgements of probable cause and explanatory relevance. Thinking \& Reasoning, 2:273-308.

P. Johnson-Laird. 1983. Mental Models : Towards a Cognitive Science of Language. Harvard University Press.

D. Kahneman. 2011. Thinking, fast and slow. Farrar, Straus, and Giroux.

Nora Kassner and H. Schütze. 2020. Negated and misprimed probes for pretrained language models: Birds can talk, but cannot fly. In ACL.

Patrick Lewis, Ethan Perez, Aleksandara Piktus, Fabio Petroni, V. Karpukhin, Naman Goyal, Heinrich Kuttler, M. Lewis, Wen tau Yih, Tim Rocktäschel, Sebastian Riedel, and Douwe Kiela. 2020. Retrievalaugmented generation for knowledge-intensive nlp tasks. In NeurIPS.

Tao Li, Vivek Gupta, Maitrey Mehta, and Vivek Srikumar. 2019. A logic-driven framework for consistency of neural models. In EMNLP.
Jiachang Liu, Dinghan Shen, Yizhe Zhang, Bill Dolan, L. Carin, and Weizhu Chen. 2021. What makes good in-context examples for GPT-3? ArXiv, $\mathrm{abs} / 2101.06804$

John W. McCarthy. 1959. Programs with common sense. In Proc. Tedding Conf. on the Mechanization of Thought Processes, pages 75-91.

Pasquale Minervini and S. Riedel. 2018. Adversarially regularising neural NLI models to integrate logical background knowledge. In CoNLL.

R. Moore. 1983. Semantical considerations on nonmonotonic logic. In IJCAI.

G. I. Parisi, Ronald Kemker, Jose L. Part, Christopher Kanan, and S. Wermter. 2019. Continual lifelong learning with neural networks: A review. Neural networks : the official journal of the International Neural Network Society, 113:54-71.

J. Pearl. 1986. Fusion, propagation, and structuring in belief networks. Artif. Intell., 29:241-288.

F. Petroni, Tim Rocktäschel, Patrick Lewis, A. Bakhtin, Yuxiang Wu, Alexander H. Miller, and S. Riedel. 2019. Language models as knowledge bases? In EMNLP.

Jonathan Pilault, Amine Elhattami, and C. Pal. 2021. Conditionally adaptive multi-task learning: Improving transfer learning in nlp using fewer parameters \& less data. In ICLR.

J. Pujara, H. Miao, L. Getoor, and William W. Cohen. 2013. Knowledge graph identification. In International Semantic Web Conference.

Abhilasha Ravichander, Eduard Hovy, Kaheer Suleman, Adam Trischler, and Jackie Chi Kit Cheung. 2020. On the systematicity of probing contextualized word representations: The case of hypernymy in BERT. In STARSEM.

Marco Tulio Ribeiro, Carlos Guestrin, and Sameer Singh. 2019. Are red roses red? Evaluating consistency of question-answering models. In $A C L$.

Adam Roberts, Colin Raffel, and Noam Shazeer. 2020. How much knowledge can you pack into the parameters of a language model? In EMNLP.

Vered Shwartz, Peter West, Ronan Le Bras, Chandra Bhagavatula, and Yejin Choi. 2020. Unsupervised commonsense question answering with self-talk. In EMNLP.

Robyn Speer, Joshua Chin, and Catherine Havasi. 2017. Conceptnet 5.5: An open multilingual graph of general knowledge. In $A A A I$.

Sanjay Subramanian, Ben Bogin, Nitish Gupta, Tomer Wolfson, Sameer Singh, Jonathan Berant, and Matt Gardner. 2020. Obtaining faithful interpretations from compositional neural networks. In $A C L$. 
Sainbayar Sukhbaatar, Arthur D. Szlam, J. Weston, and R. Fergus. 2015. End-to-end memory networks. In NeurIPS.

Oyvind Tafjord and Peter Clark. 2021. Generalpurpose question-answering with Macaw. arXiv, $\mathrm{abs} / 2109.02593$.

Alon Talmor, Oyvind Tafjord, Peter Clark, Yoav Goldberg, and Jonathan Berant. 2020. Leap-of-thought: Teaching pre-trained models to systematically reason over implicit knowledge. In NeurIPS.

Xiaojin Zhu. 2015. Machine teaching: An inverse problem to machine learning and an approach toward optimal education. In AAAI. 


\section{Appendix: BeliefBank: Adding Memory to a Pre-Trained Language Model for a Systematic Notion of Belief}

\section{A Selecting Constraint Rules from ConceptNet}

As described in Section 5.1, positive implication (constraint) rules were manually gathered from ConceptNet (Speer et al., 2017). First, we identified 121 general concepts of interest, e.g., "mammal", choosing concepts with high occurrence (> 100 times) in ConceptNet, avoiding significantly ambiguous terms (e.g., "bat"), and filtering out plurals and obscure concepts. For these entities, we then collected all ConceptNet facts involving 6 relations: IsA, HasA, MadeOf, PartOf, HasProperty, and CapableOf, and re-expressed them as constraints. For example, the ConceptNet triple (dog, HasA, tail) gives rise to the constraint " $\mathrm{X}$ is a dog" $\rightarrow$ "X has a tail." (Triples are converted into English sentences using simple templates). We then manually filter theses constraints for factual correctness. We also add weaker, disjunctive constraints in the backwards direction, e.g., "X has a tail" $\rightarrow$ "X is a dog" $O R$ " $\mathrm{X}$ is a cat" $O R$.... for all entities with tails. (These backwards rules discourage the trivial solution that everything is false.) Finally, two hyperparameters for weights on forward and backwards rules are set by automatic calibration (Section 5.2).

\section{B Experimental results in table form}

Tables 1 and 2 contain the numerical data for the experimental results plotted in Figure 3. 


\begin{tabular}{lcccccccccc}
\hline Accuracy (F1) after batch $\rightarrow$ & 1 & 2 & 3 & 4 & 5 & 6 & 7 & 8 & 9 & 10 \\
\hline Raw model & 69.2 & 70.0 & 70.0 & 69.3 & 69.3 & 69.5 & 69.2 & 69.0 & 69.1 & 69.3 \\
OnTopic-FB & 69.2 & 74.3 & 76.0 & 76.4 & 77.0 & 77.3 & 77.5 & 77.5 & 77.8 & 77.8 \\
Relevant-FB & 69.2 & 73.8 & 75.1 & 75.8 & 76.8 & 77.2 & 77.7 & 78.4 & 78.9 & 79.5 \\
Constraints & 72.3 & 75.2 & 76.8 & 78.3 & 80.1 & 81.6 & 82.4 & 83.3 & 84.9 & 85.8 \\
Relevant-FB + Constraints & 72.4 & 78.5 & 80.5 & 82.1 & 83.6 & 84.2 & 85.1 & 86.1 & 86.7 & 86.6 \\
\hline
\end{tabular}

Table 1: Experimental results for accuracy (F1), as plotted in Figure 3, here shown in tabular form.

\begin{tabular}{lcccccccccc}
\hline Consistency $(1-\tau)$ after batch $\rightarrow$ & 1 & 2 & 3 & 4 & 5 & 6 & 7 & 8 & 9 & 10 \\
\hline Raw model & 73.2 & 73.7 & 74.0 & 72.8 & 72.4 & 72.3 & 72.3 & 72.2 & 72.4 & 72.5 \\
OnTopic-FB & 73.2 & 72.9 & 73.6 & 73.3 & 73.5 & 72.6 & 72.5 & 72.6 & 72.5 & 72.3 \\
Relevant-FB & 73.2 & 77.8 & 79.3 & 80.1 & 80.6 & 79.7 & 80.1 & 80.5 & 80.8 & 81.0 \\
Constraints & 98.2 & 97.1 & 96.5 & 96.2 & 96.3 & 96.4 & 96.1 & 96.1 & 96.0 & 96.0 \\
Relevant-FB + Constraints & 98.8 & 98.2 & 97.6 & 97.8 & 97.9 & 97.7 & 97.3 & 97.2 & 97.1 & 97.0 \\
\hline
\end{tabular}

Table 2: Experimental results for consistency $(1-\tau)$, as plotted in Figure 3, here shown in tabular form. 